\title{
Perfect Edge Domination: Hard and Solvable Cases
}

\author{
Min Chih $\operatorname{Lin}^{1}$ \\ Veronica A. Moyano ${ }^{1}$ \\ Vadim Lozin ${ }^{2}$ \\ Jayme L Szwarcfiter ${ }^{3}$ \\ ${ }^{1}$ Universidad de Buenos Aires \\ Argentina \\ oscarlin@dc.uba.ar, vmoyano@ic.fcen.uba.ar \\ ${ }^{2}$ University of Warwick \\ United Kingdom \\ v.lozin@warwick.ac.uk \\ ${ }^{3}$ Universidade Federal do Rio de Janeiro, and \\ Instituto Nacional de Metrologia, Qualidade e Tecnologia \\ Brasil \\ jayme@nce.ufrj.br
}

\begin{abstract}
Let $G$ be an undirected graph. An edge of $G$ dominates itself and all edges adjacent to it. A subset $E^{\prime}$ of edges of $G$ is an edge dominating set of $G$, if every edge of the graph is dominated by some edge of $E^{\prime}$. We say that $E^{\prime}$ is a perfect edge dominating set of $G$, if every edge not in $E^{\prime}$ is dominated by exactly one edge of $E^{\prime}$. The perfect edge dominating problem is to determine a least cardinality perfect edge dominating set of $G$. For this problem, we describe two NP-completeness proofs, for the classes of claw-free graphs of degree at most 3, and for bounded degree graphs, of maximum degree at most $d \geq 3$ and large girth. In contrast, we prove that the problem admits an $O(n)$ time solution, for cubic claw-free graphs. In addition, we prove a complexity dichotomy theorem for the perfect edge domination problem, based on the results described in the paper. Finally, we describe a linear time algorithm for finding a minimum weight perfect edge dominating set of a $P_{5}$-free graph. The algorithm is robust, in the sense that, given an arbitrary graph $G$, either it computes a minimum weight perfect edge dominating set of $G$, or it exhibits an induced subgraph of $G$, isomorphic to a $P_{5}$.
\end{abstract}

\section{Introduction}

Edge domination problems have been the focus of considerable attention, in the last few years. Among the relevant variations of these problems are the perfect edge domination and efficient edge domination. In the former variation, each edge not in the dominating set is dominated by exactly one edge, while in the latter, every edge of the graph is so dominated. The problems consist in determining such dominating sets, of minimum cardinality or minimum total weight of their edges. Most of the known results, so far concerned the efficient edge dominating case (also known as dominating induced matching), for instance see 2, 3, 4, 6, 8, 9, 10, 11, 12, 13, 14, 15, 16, 17. These two types of dominations may lead to problems of a quite different nature. To start with, an efficient edge dominating set of a graph may not exist, while it necessarily exists for the perfect domination. Furthermore, there are important differences concerning the complexity status of the problems for classes of graphs although both of them are hard, in general. In fact, perfect edge domination problems seem to be not easier to tackle than 
efficient edge domination. On the other hand, if a graph contains an efficient edge dominating set then such set is also a minimum cardinality perfect edge dominating set [7, [15]. The corresponding decision problems, for the cardinality version, are therefore as follows.

\author{
EFFICIENT EDGE DOMINATION \\ INPUT: Graph $G$ \\ QUESTION: Does $G$ contain an efficient edge dominating set?
}

\title{
PERFECT EDGE DOMINATION
}

INPUT: Graph $G$, integer $p$

QUESTION: Does $G$ contain a perfect edge dominating set $\mathrm{S}$ of size $\leq p$ ?

Both problems are known to be NP-complete [8] and [15, respectively.

In the present paper, we consider perfect edge domination. The main proposed results are as follows:

- A NP-hardness proof for the cardinality version of perfect edge domination in claw-free graphs of degree at most 3.

- A linear time solution for weighted (with possibly negative weights) perfect edge domination in cubic claw-free graphs.

- A NP-hardness proof for the cardinality version of perfect edge domination in bounded-degree graphs of large girth. The proof also implies NP-hardness for $r$-regular graphs, $r \geq 3$.

- A complexity dichotomy theorem which establishes NP-hardness for the perfect edge domination problem in any class of bounded degree graphs, defined by forbidding one general graph $H$. The only exception is when $H$ is a set of disjoint paths, in which case the corresponding perfect edge domination problem admits a polynomial time solution.

- A robust linear time algorithm for solving weighted perfect edge domination problem of $P_{5}$-free graphs. That is, in linear time, the algorithm either finds a minimum weight edge dominating set of the graph, or exhibits an induced $P_{5}$.

We remark that the above problems have already been solved for efficient edge domination. In contrast with the perfect edge domination hardness proposed in the present paper, Cardoso, Koperlainen and Lozin [6] have described an $O\left(n^{2}\right)$ time algorithm for finding an efficient edge dominating set, for general claw-free graphs. More recently, the corresponding weighted problem has been solved in $O(n)$ time, by Lin, Mizrahi and Szwarcfiter [12. Moreover, Hertz, Lozin, Ries, Zamaraev and de Werra 9 have shown that the efficient edge domination problem can still be solved in polynomial time for graphs containing no long claws. Finally, for bounded degree graphs, Cardoso, Cerdeira, Delorme and Silva [4 have shown that efficient edge domination problem is NP-complete for $r$-regular graphs for $r \geq 3$.

Known results to the authors for the perfect edge domination problem are as follows. Lu, Ko and Tang [15] proved the problem is NP-complete for bipartite graphs. As for polynomial time solvable cases, there are linear time algorithms also described by $\mathrm{Lu}$, Ko and Tang, for generalized series-parallel graphs and chordal graphs. In addition, there is a linear time algorithm for circular-arc graphs, by Lin, Mizrahi and Szwarcfiter 13. All of these algorithms solve the weighted perfect edge domination problem.

The structure of the paper is as follow. Section 2 describes the terminology employed. Section 3 contains the NP-completeness proof of the perfect edge domination problem for claw-free graphs of degree at most three. In Section 4 we show that the weighted perfect edge domination problem is solvable in linear time for cubic claw-free graphs. In Section 5 we prove NP-hardness of the perfect edge domination problem in bounded degree graphs even restricted to those of large girth. This result implies the NP-hardness for $r$-regular graphs with $r \geq 3$. Section 6 contains the dichotomy theorem for the complexity of the perfect edge domination problem for bounded degree $H$-free graphs. When $H$ is a disjoint union of paths the problem is solvable in polynomial 
time, otherwise it remains NP-hard. Section 7 is dedicated to the case $H=P_{k}$. For $P_{5}$-free graphs we present a robust algorithm which solves the weighted perfect edge domination problem in linear time.

\section{Preliminaries}

Let $G$ be an undirected graph with no loops or multiple edges. The vertex and edge sets of $G$ are denoted by $V(G)$ and $E(G)$, respectively, $|V(G)|=n$. For $v, w$, adjacent vertices of $G$, write $v w$ to denote the edge incident to $v$ and $w$. For $v \in V(G)$, let $N(v)=\{w \in V(G) \mid v, w$ are adjacent $\}$, and $N[v]=N(v) \cup\{v\}$. The edges $e$ and $e^{\prime}$ are adjacent if they are incident to a common vertex. For $e \in E(G)$, let $N^{\prime}(e)=\left\{e^{\prime} \in E(G) \mid e, e^{\prime}\right.$ are adjacent $\}$, and $N^{\prime}[e]=N^{\prime}(e) \cup\{e\}$. For $V^{\prime} \subseteq V(G)$, denote by $G\left[V^{\prime}\right]$ the subgraph induced in $G$ by $V^{\prime}$. Similarly, for $E^{\prime} \subseteq E(G)$, denote by $G\left[E^{\prime}\right]$ the subgraph of $G$ having exactly the edges of $E^{\prime}$ without isolated vertices. Each edge $e \in E(G)$ may be assigned a real value, called the weight of $e$. The girth of $G$ is the length of a shortest cycle contained in $G$. If $G$ is an acycle graph the girth is defined to be infinity.

A vertex $v \in V(G)$ dominates itself and any other vertex adjacent to it. A subset of vertices $X \subseteq V(G)$ is a (vertex) dominating set if every vertex of $G$ is dominated by some vertex of $X$. An edge vw $\in E(G)$ dominates itself and any other edge adjacent to it. A subset of edges $E^{\prime} \subseteq E(G)$ is a perfect edge dominating set (PEDS) of $G$, if every edge of $E(G) \backslash E^{\prime}$ is dominated by exactly one edge of $E^{\prime}$. On the other hand if every edge of $E(G)$ is dominated exactly once by $E^{\prime}$ then $E^{\prime}$ is an efficient edge dominating set (EEDS), also called a dominating induced matching. The cardinality perfect (efficient) edge domination problem is to determine the perfect (efficient) edge dominating set of $G$ of least cardinality. The corresponding weighted problems are defined replacing minimum cardinality by minimum sum of weights of the dominating edges.

Let $P \subseteq E(G)$ be a perfect edge dominating set of a connected graph $G$. Then $P$ defines a 3 -coloring of the vertices of $G$, as below:

- black vertices: Those having at least two incident edges of $P$. We denote this subset of vertices by $B$.

- yellow vertices: Those which are incident to exactly one edge of $P$. We denote this subset of vertices by $Y$.

- white vertices: The ones not incident to any edge of $P$. We denote this subset of vertices by $W$.

We call $(B, Y, W)$ the 3-coloring associated to $P$.

Observation $1 W$ is an independent set.

Observation 2 Pendant vertices (vertices of degree 1) in $G \backslash W$ are exactly the yellow vertices.

Observation 3 White vertices have only yellow neighbors.

Observation 4 Every vertex of an induced $K_{t}$ with $t \geq 4$ must be black.

Observation 5 Every induced triangle has (i) three black vertices or (ii) two yellow vertices and one white vertex.

It is straightforward to see that any 3 -coloring $(B, Y, W)$ of the vertices of $G$, that verifies the conditions of Observations 1, 2 and 3, is necessarily associated to some perfect edge dominating set of $G$.

On the other hand, an efficient edge dominating set is clearly a perfect edge dominating set. In this case, the set $B$ is empty. The latter implies that the subset induced by $Y$ is an induced matching of $G$. 


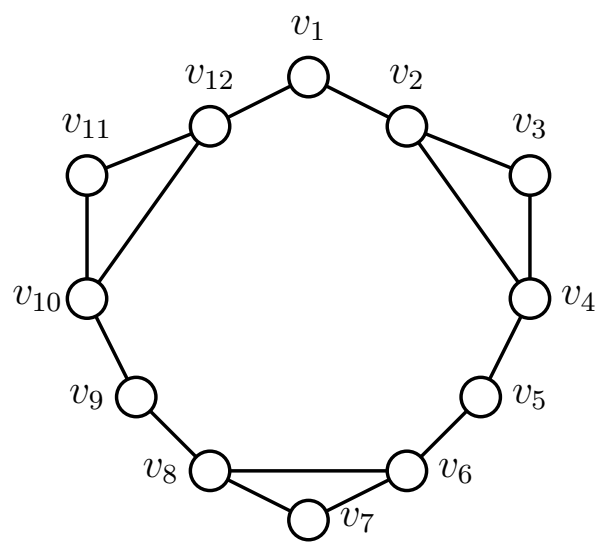

Figure 1: The shield graph

Clearly, the converse is not true. When $B \neq \emptyset, P$ is not an efficient edge dominating set. In particular, $E(G)$ is a perfect edge dominating set, called the trivial perfect edge dominating set, when $W=\emptyset$. Any perfect edge dominating set $P$ which is neither trivial nor an efficient edge dominating set is called proper perfect edge dominating set because $W, B, Y \neq \emptyset$. Similarly, $(B, Y, W)$ is then called a proper coloring.

\section{Claw-free graphs of degree at most three}

In this section, we prove NP-completeness of the perfect edge domination problem, for claw-free graphs, of degree at most three. We recall that the efficient edge domination problem can be solved in polynomial time for general claw-free graphs [6, 12]. However, for $r$-regular graphs, fixed $r \geq 3$, efficient edge domination problem is also NP-complete [4.

Theorem 6 [4] For arbitrary fixed $r \geq 3$, deciding on the existence of efficient edge dominating sets on $r$-regular graphs is NP-complete.

Let $G$ be an $r$-regular graph with $n$ vertices. Then the number of its edges is exactly $\frac{r n}{2}$. Suppose $G$ admits some efficient edge dominating set $D$. It will be useful to determine $|D|$. Let $(B=\emptyset, Y, W)$ be the 3-coloring associated to $D$. It is easy to see, $|Y|=2|D|,|W|=n-2|D|, \frac{r n}{2}-|D|=r|W|=r(n-2|D|)=r n-2 r|D|$ and $\frac{r n}{2}-|D|=(r-1)|Y|=(r-1) 2|D|=2 r|D|-2|D|$ which implies $r n-2 r|D|=2 r|D|-2|D|$ and $r n=4 r|D|-2|D|=(4 r-2)|D|$. Hence, $|D|=\frac{r n}{4 r-2}$.

Now, let us turn to the perfect edge domination problem. Start by determining the possible perfect edge dominating sets for the graph of Figure 1.

Lemma 7 The only perfect edge dominating sets of the shield graph are the following cases:

(i) $\left\{v_{2} v_{4}, v_{6} v_{8}, v_{10} v_{12}\right\}$ (Figure 圆. Actually, it is an efficient edge dominating set.

(ii) $\left\{v_{3} v_{4}, v_{6} v_{7}, v_{9} v_{10}, v_{10} v_{11}, v_{11} v_{12}, v_{12} v_{1}, v_{10} v_{12}\right\},\left\{v_{2} v_{3}, v_{5} v_{6}, v_{6} v_{7}, v_{7} v_{8}, v_{8} v_{9}, v_{6} v_{8}, v_{11} v_{12}\right\}$ and $\left\{v_{1} v_{2}, v_{2} v_{3}, v_{3} v_{4}\right.$, $\left.v_{4} v_{5}, v_{2} v_{4}, v_{7} v_{8}, v_{10} v_{11}\right\}$ (Figure 3 ). They are proper perfect edge dominating sets and are symmetrical to each other.

(iii) The trivial perfect edge dominating set. (Figure 4). 


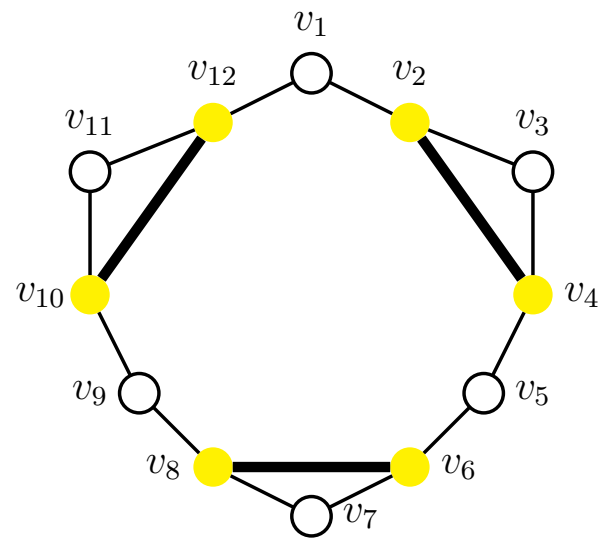

Figure 2: The minimum PEDS which is a EEDS
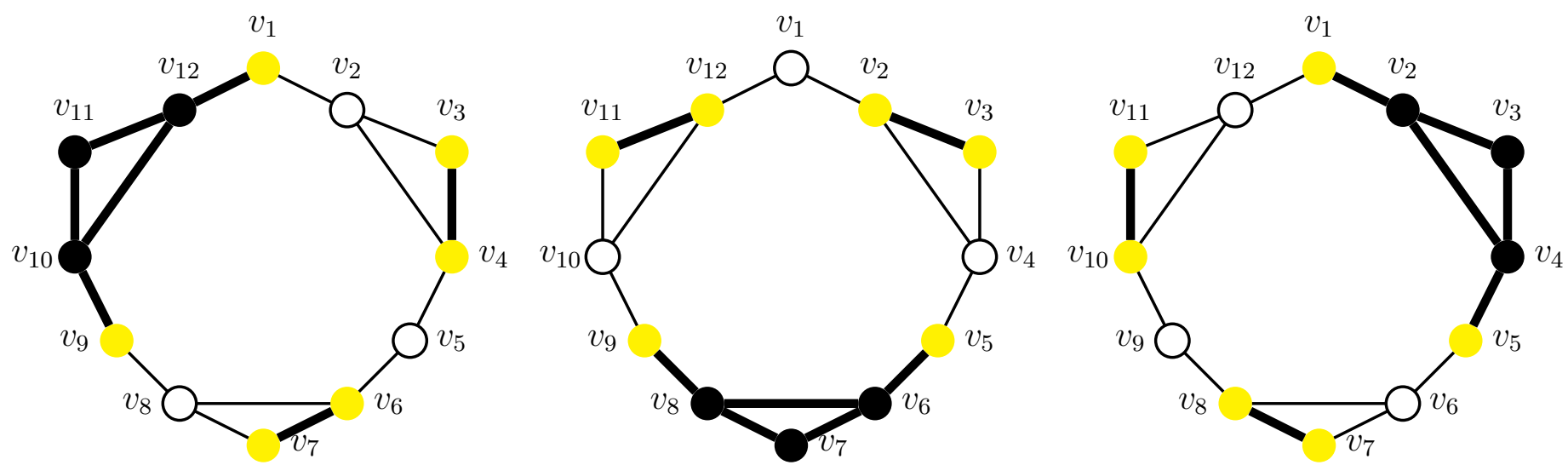

Figure 3: Proper PEDS

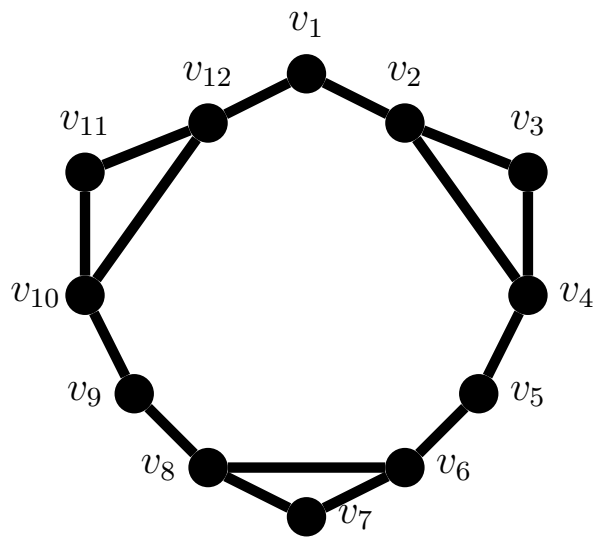

Figure 4: The trivial PEDS 
Proof: Clearly, all edges subsets described in (i), (ii) and (iii) are perfect edge dominating sets of the shield graph. Let $P$ be a perfect edge dominating set of such a graph and $(B, Y, W)$, the 3 -coloring associated to $P$. Consider the colors of $v_{3}, v_{7}$ and $v_{11}$.

- The vertices $v_{3}, v_{7}, v_{11} \in B$. By Observation $5, v_{2}, v_{4}, v_{6}, v_{8}, v_{10}$ and $v_{12}$ are also black vertices. $v_{1}, v_{5}$ and $v_{9}$ can be neither white vertices (Observation 3) nor yellow vertices (Observation 2). Hence, all vertices have black colors which implies $P$ to be the trivial perfect edge dominating set described in (iii).

- Some of these vertices are white. Without loss of generality, $v_{3}$ has white color. By Observation $5 v_{2}$ and $v_{4}$ are yellow vertices. By observation 2, $v_{1}$ and $v_{5}$ must be white vertices. And by Observation 3, $v_{12}$ and $v_{6}$ are yellow vertices. Clearly, by Observation 5, exactly one of $v_{7}$ or $v_{8}$ is yellow vertex and the other is white. Suppose that $v_{7} \in Y$ and $v_{8} \in W$. Then $v_{9} \in Y$ (Observation 3) and $v_{10} \notin W$ (Observation 22). As $v_{10}$ has two yellow neighbors $\left(v_{9}\right.$ and $\left.v_{12}\right)$, by Observation 2, $v_{10}$ is a black vertex. Therefore, the condition of Observation 5 does not hold for the triangle $\left\{v_{10}, v_{11}, v_{12}\right\}$, a contradiction. Consequently, $v_{8} \in Y$ and $v_{7} \in W$. Symmetrically, using exactly the same argument, we conclude that $v_{10} \in Y$ and $v_{11} \in W$. Finally, $v_{9}$ is a white vertex by Observation 2 and clearly $P$ is the efficient edge dominating set described in (i).

- One of these vertices is yellow. Without loss of generality, $v_{3}$ has yellow color. By Observation 5 , exactly one of $v_{2}$ or $v_{4}$ is a yellow vertex and the other is a white vertex. Again, without loss of generality $v_{2} \in Y$ and $v_{4} \in W$. By Observation 3, $v_{5} \in Y$ and by Observation 2, $v_{1} \in W$. By Observation 3. $v_{12}$ must be yellow vertex. Clearly, by Observation [5, exactly one of $v_{10}$ or $v_{11}$ is yellow vertex and the other is white. Suppose that $v_{10} \in Y$ and $v_{11} \in W$, then $v_{9} \in W$ (Observation 2) and $v_{8} \in Y$ (Observation 3). On the other hand, as $v_{5} \in Y$, by Observation 2 $v_{6} \notin W$ and has two yellow neighbors $\left(v_{5}\right.$ and $\left.v_{8}\right)$ which implies by Observation 2, $v_{6} \in B$. Hence, the condition of Observation 5 does not hold for the triangle $\left\{v_{6}, v_{7}, v_{8}\right\}$ again a contradiction. Consequently, $v_{11} \in Y$ and $v_{10} \in W$. By Observation 3, $v_{9} \in Y$ and by Observation 2, $v_{6}, v_{8} \notin W$. As $v_{6}\left(v_{8}\right)$ has two non-white neighbors, its color must be black. Finally by Observation $5 . v_{7} \in B$, and clearly $P$ is the second perfect edge dominating set described in (ii). Making the other choices of options, leads to the other two perfect edge dominating sets of (ii). $\triangle$

We employ an operation that replaces certain vertices by the shield graph.

Let $G$ be a graph having a vertex $v$ of degree 3 , and $x, y$ and $z$, the neighbors of $v$. The magnification of $v$ is the operation that replaces $v$ by a shield $S_{v}$ and replaces the edges $x v, y v, z v$ by $x v_{3}, y v_{7}, z v_{11}$ (we call $v_{3}, v_{7}$ and $v_{11}$ the contact vertices of $S_{v}$ ). The magnification of a cubic graph $G, \mathcal{M}(G)$, is the graph obtained applying magnification to all its vertices. The following claim is obvius.

Claim $1 \mathcal{M}(G)$ is claw-free and has degree at most 3.

If $G$ has $n$ vertices (implying that $G$ has $\frac{3 n}{2}$ edges) then $\mathcal{M}(G)$ has $12 n$ vertices and $15 n+\frac{3 n}{2}=\frac{33 n}{2}$ edges.

We relate the cardinality of an efficient edge dominating set of $G$ to that of a perfect edge dominating set of $\mathcal{M}(G)$.

Theorem 8 Let $G$ be a cubic graph with n vertices and $\mathcal{M}(G)$ the magnification of $G$. Then $G$ admits some efficient edge dominating set $D$ if and only if $\mathcal{M}(G)$ has a perfect edge dominating set $P$ of size at most $\frac{57 n}{10}$.

Proof: Assume $G$ admits some efficient edge dominating set $D$ with $|D|=\frac{3 n}{10}$. Let $Y_{D}$ the subset of vertices incident to some edge of $D$ and $W_{D}=V \backslash Y_{D}$. It is clear that $\left|Y_{D}\right|=\frac{3 n}{5}$ and $\left|W_{D}\right|=\frac{2 n}{5}$. Next, we will describe how to construct a perfect edge dominating set $P$ of $\mathcal{M}(G)$ by choosing appropriately its associated 3-coloring $(B, Y, W)$. As the vertices of $\mathcal{M}(G)$ are partitioned into $n$ different shields $S_{v}$. we choose locally the coloring of each shield $S_{v}$, then we check if the total coloring is valid. If $v \in W_{D}$ then its corresponding shield $S_{v}$ takes the coloring described in Figure 2. Otherwise, $v \in Y_{D}$ and $v$ has exactly 2 white neighbors and one neighbor $v^{\prime}$ in $G$. In this case, such $S_{v}$ and $S_{v^{\prime}}$ should take some coloring described in Figure 3 in such a way that the edge connecting both shields in $\mathcal{M}(G)$ has both extreme vertices with black color. Clearly, this can be achieved. This 
coloring is valid because the partial coloring in each shield is valid and every edge that connects two shields has both extreme vertices with black color or one with white color and the other with yellow color. Now, we examine the number of edges of this perfect edge dominating set $P$. Every shield $S_{v}$ contributes 3 edges if $v$ is white and 7 edges if $v$ is yellow. The number of edges that belong to $P$ and connect two shields is exactly the number of edges in $D$. Consequently, the total number of edges of $P$ is $3\left|W_{D}\right|+7\left|Y_{D}\right|+|D|=\frac{6 n}{5}+\frac{21 n}{5}+\frac{3 n}{10}=\frac{57 n}{10}$ as required.

Conversely, suppose $\mathcal{M}(G)$ has a perfect edge dominating set $P$ of size at most $\frac{57 n}{10}$. Let $(B, Y, W)$ be the associated 3-coloring of $P$. We examine locally the coloring of each shield $S_{v}$. If some contact vertex has white color, then using the same argument as in the proof of Lemma 7 all contact vertices have white color and there are exactly 3 edges of $P$ within $S_{v}$. In this case, call $S_{v}$ a white shield. Similarly, if some contact vertex has yellow color, there are exactly 2 contact vertices with yellow color, the other contact vertex is black and there are exactly 7 edges of $P$ within $S_{v}$. In this case, call $S_{v}$ is a yellow shield. The third possibility is that all contact vertices have black color and all 15 edges of $S_{v}$ belong to $P$. We call $S_{v}$ a black shield. Denote by $b, y, w$ the numbers of black, yellow and white shields, respectively. Clearly, every white shield is adjacent to exactly 3 yellow shields (two shields are adjacent if there is some edge between their contact vertices), and every yellow shield is adjacent to two white shields and to one non-white shield. It implies that $3 w=2 y$. This is the number of edges that connect two different shields and do not belong to $P$. As the total number of edges of $\mathcal{M}(G)$ is $\frac{33 n}{2}$ and $|P| \leq \frac{57 n}{10}$, the number of edges that do not belong to $P$ is $12 w+8 y+3 w=12 w+12 w+3 w=27 w \geq \frac{108 n}{10}$. That is $w \geq \frac{2 n}{5}$. Hence, $y \geq \frac{3 n}{5}$ and $b=0$. Moreover, $w=\frac{2 n}{5}$ and $y=\frac{3 n}{5}$. We can define the color of each vertex $v$ of $G$ as the color of $S_{v}$. This coloring corresponds to an efficient edge dominating set of $G$. $\triangle$

The next corollary is a consequence of Theorems 6 and 8 .

Corollary 9 The minimum perfect edge domination problem for claw-free graphs of degree at most 3 is NPhard.

Proof: Clearly, checking if a subset of edges is a perfect edge dominating set of at most certain size can be done in polynomial time. This implies the problem is $\mathcal{N} P$. To prove the completeness, we can apply the reduction from efficient edge domination problem for cubic graphs which is NP-complete by Theorem 6. Take any instance of this problem. The input is a cubic graph $G$ with $n$ vertices. We transform $G$ to $\mathcal{M}(G)$ which is claw-free and has degree at most 3. This can be done in polynomial-time. By Theorem $8, G$ has an efficient edge dominating set if only if $\mathcal{M}(G)$ has a perfect edge dominating set of size at most $\frac{57 n}{10}$. Therefore, the proof is complete. $\triangle$

\section{Cubic claw-free graphs}

In this section, we show that the weighted version of the perfect edge domination problem can be solved in linear time for cubic claw-free graphs. Without loss of generality, we consider only connected graphs.

The first lemma concerns efficient edge dominating sets of claw-free graphs.

Lemma 10 [12] There is an algorithm of complexity $O(n)$ which finds the least weight efficient edge dominating set of a claw-free graph $G$, or reports that $G$ does not admit efficient edge dominating sets.

The following observation is useful.

Observation 11 A graph $G$ is a cubic claw-free graph if and only if $G$ is cubic and every vertex of it is contained in some triangle.

The second lemma concerns proper perfect edge dominating sets of connected graphs where every vertex is contained in some triangle. 
Lemma 12 Let $G$ be a connected graph where every vertex is contained in some triangle. Then $G$ admits no proper perfect edge dominating set.

Proof: Suppose the contrary and let $P$ be a proper perfect edge dominating set of $G$, and $(B, Y, W)$ its corresponding coloring of the vertices of $G$. The idea is to show that every vertex of $G$ has black color which implies that $P$ is the trivial perfect edge dominating set, which is a contradiction. Since $P$ is proper, $G$ contains some black vertex $v$. By the hypotesis, $v$ is contained in at least one triangle of $G$. Clearly, any pair of adjacent neighbors of $v$ must have black color by Observation [5. We can apply iteratively the same reasoning to each new considered black vertex and we call this procedure as black propagation. If every vertex of $G$ has been considered, we are done. Suppose there is some unconsidered vertex. As $G$ is connected, there must be an edge $u w$ in $G$ such that $u$ has been considered and $w$ has not. It is clear that $u$ and $w$ do not have a common neighbor. By the hypotesis, there is a triangle $C^{\prime}$ containing $w$. Since $u$ has black color, by Observation 3, $w$ cannot be a white vertex. By Observation 5, $w$ must have some neighbor with non-white color in $C^{\prime}$. But $w$ has already a black neighbor $u$ which is not in $C^{\prime}$. Therefore, $w$ has black color by Observation 2, Again, we can apply the black propagation to $w$. Repeating iteratively the same argument, we conclude that all vertices of $G$ must be black, and therefore $G$ cannot contain a proper edge dominating set. $\triangle$

The algorithm follows immediately from the two above lemmas and the Observation [11, Let $G$ be a connected cubic claw-free graph, whose edges have been assigned weights, possibly negative. By Lemma 12 it does not contain proper perfect edge dominating sets. Then first, we apply the algorithm described in 12 for claw-free graphs. If $G$ contains an efficient edge dominating set then the latter algorithm finds the least weighted of such sets, and the minimum between this set and the trivial perfect edge dominating set $E(G)$ is the answer of our algorithm. Otherwise, $G$ does not contain an efficient edge dominating set, and therefore the only perfect edge dominating set of $G$ is $E(G)$ and this is the answer of the algorithm.

By recalling that $|E(G)|=\frac{3 n}{2}$, the complexity of the algorithm is therefore $O(n)$.

This linear time algorithm can be extended easily to claw-free graphs where every degree two vertex has two adjacent neighbors. Because in this case, every vertex is in some triangle except for pendant vertices. We can see that Lemma 12 is still valid for any connected component of these graphs. We know that pendant vertices never have black colors by Observation 2, If there is some black vertex in the connected component, then all vertices of this component are colored black, except the pendant vertices. Therefore, every such pendant vertex has a black neighbor if the component is not exactly $K_{2}$. In any case, every pendant vertex must be colored yellow. Consequently, there are no white vertices and the lemma is true.

\section{$5 \quad$ Bounded degree graphs of large girth}

In this section, we prove NP-hardness of the perfect edge domination problem in bounded degree graphs even restricted to those of large girth.

The following additional notation is employed. For a non negative integer $k$, and an edge $e=v w \in E(G)$, where $v, w \in V(G)$, the $k$-subdivision of $e$ is the operation that replaces $e$ by a $(k+1)$-path, denoted by $S_{k}(e)$, formed by edges $e_{0}, \ldots, e_{k}$, such that all its $k$ internal vertices are newly inserted vertices, each of degree 2 , while $e_{0}$ is incident to $v$ and $e_{k}$ incident to $w$. In general, for $E^{\prime} \subseteq E, S_{k}\left(E^{\prime}\right)$ is the set of the $k$-subdivisions of the edges of $E^{\prime}$, while $S_{k}(G)$ denotes the $k$-subdivision graph of $G$, the one obtained by subdividing all its edges. In such a graph, the extreme vertices of each edge $e \in E(G)$ become the extremes of the paths $S_{k}(e)$ and are called extremes of $S_{k}(G)$. We omit the subscript from $S$ when there is no ambiguity.

Throughout this section, $G$ denotes an $r$-regular graph, and $S(G)$ the $3 k$-subdivision of $G$, for some integer $k \geq 0$.

Lemma 13 Let $e=v w \in E(G)$ and $P$ be a perfect edge dominating set of $S(G)$. Then

1. $|S(e) \cap P| \geq k$. 


$$
\text { 2. }|S(e) \cap P|=k \Rightarrow e_{0}, e_{3 k} \notin P,|\{v, w\} \cap W|=1 \text { and }|\{v, w\} \cap Y|=1 \text {. }
$$

Proof: Note that Lemma 13 holds for $k=0$ since (1.) is trivially true and (2.) holds because $e=e_{0}=e_{k}$ is dominated by $P$. For $k \geq 1$ since $P$ is a perfect edge dominating set, at least one in every three consecutive edges of $S(e)$ must belong to $P$. Observe the following subsets of edges $\left\{e_{1}, e_{2}, e_{3}\right\},\left\{e_{4}, e_{5}, e_{6}\right\}, \ldots,\left\{e_{3 k-2}, e_{3 k-1}, e_{3 k}\right\}$. Each of them has at least one edge of $P$ and we can conclude that $|S(e) \cap P| \geq k$. If some of them has at least two edges of $P$ then $|S(e) \cap P| \geq k+1$. Otherwise, every one has exactly one edge of $P$. Easily, we can determine these edges knowing which edge of $\left\{e_{1}, e_{2}, e_{3}\right\}$ belongs to $P$. The three possibilities are: (i) $e_{1}, e_{4}, \ldots, e_{3 k-2}$ (ii) $e_{2}, e_{5}, \ldots, e_{3 k-1}$ and (iii) $e_{3}, e_{6}, \ldots, e_{3 k}$.

Next, we assume that $|S(e) \cap P|=k$ and we consider the alternatives:

1. $e_{0} \in P$

Then $|S(e) \cap P|=\left|\left\{e_{0}\right\}\right|+\left|\left\{e_{1}, e_{2}, e_{3}\right\} \cap P\right|+\ldots+\left|\left\{e_{3 k-2}, e_{3 k-1}, e_{3 k}\right\} \cap P\right| \geq 1+k$ which is a contradiction.

2. $e_{3 k} \in P$

Using similar argument of Alternative 1, we can conclude that $|S(e) \cap P| \geq k+1$. Again, a contradiction.

3. $e_{0}, e_{3 k} \notin P$

Examine the further alternatives:

(a) $v, w \in W$ :

Clearly, $e_{1}, e_{3 k-1} \in P$. Hence, some of the subsets $\left\{e_{1}, e_{2}, e_{3}\right\},\left\{e_{4}, e_{5}, e_{6}\right\}, \ldots,\left\{e_{3 k-2}, e_{3 k-1}, e_{3 k}\right\}$ has at least two edges of $P$ and implies that $|S(e) \cap P| \geq k+1$. This is a contradiction.

(b) $v, w \in Y$ :

Then $e_{1}, e_{3 k-1} \notin P$ because $e_{0}\left(e_{3 k}\right)$ is dominated by some edge incident to $v(w)$. Furthermore, $e_{2}, e_{3 k-2} \in P$ (to dominate $e_{1}$ and $e_{3 k-1}$, respectively). Again, some of the subsets $\left\{e_{1}, e_{2}, e_{3}\right\}$, $\left\{e_{4}, e_{5}, e_{6}\right\}, \ldots,\left\{e_{3 k-2}, e_{3 k-1}, e_{3 k}\right\}$ has at least two edges of $P$ implying that $|S(e) \cap P| \geq k+1$ and leading to a contradiction.

(c) $v \in B$ or $w \in B$ :

Then $e_{0} \in P$ or $e_{3 k} \in P$ which is a contradiction to the assumption.

(d) One of $v, w$ is $W$ and the other one is $Y$. This is the only situation where $|S(e) \cap P|=k$ can occur.

$\triangle$

The next lemma describes a lower bound for the size of a perfect edge dominating set of $S(G)$.

Lemma 14 Let $e \in E(G)$ and $P$ be a perfect edge dominating set of $S(G)$. Then

1. $\left|S\left(N^{\prime}[e]\right) \cap P\right| \geq 2 r k-k+1$

2. $|P| \geq \frac{n r}{2(2 r-1)} \cdot(2 r k-k+1)$

Proof: By Lemma 13, it follows that $|S(e) \cap P| \geq k$, for any $e \in E(G)$. Since $G$ ia an $r$-regular graph, $\left|N^{\prime}[e]\right|=2 r-1$. Consequently, $\left|S\left(N^{\prime}[e]\right) \cap P\right| \geq(2 r-1) k$. In addition, also by Lemma 13. $|S(e) \cap P|=k$ implies that an extreme vertex $v$ of $e$ belongs to $W$, the other extreme $w \in Y$, and the extreme edge $e_{3 k}$ of $S(e)$, incident to $w$, is not in $P$. In order to attain $\left|S\left(N^{\prime}[e]\right) \cap P\right|=(2 r-1) k$, all $2 r-1$ edges of $N^{\prime}[e]$ must satisfy the latter condition. However, the yellow extreme vertex of $e$ must have some incident edge $e^{\prime \prime} \in P$ and this edge $e^{\prime \prime}$ belongs to some $S\left(e^{\prime}\right), e^{\prime} \in E(G)$. Clearly, $e^{\prime} \in N^{\prime}(e)$ and according to Lemma 13. $\left|S\left(e^{\prime}\right) \cap P\right| \geq k+1$. Consequently, the bound is refined as $S\left(N^{\prime}[e] \cap P\right) \geq(2 r-2) k+k+1=2 r k-k+1$, proving the first part of the lemma.

To prove the second part, we remind that in each edge neighborhood $N^{\prime}[e]$ of $G, 2 r-2$ of the edges of $S\left(N^{\prime}[e]\right)$ contain at least $k$ edges of $P$, while one edge of $N^{\prime}[e]$ corresponds in $S\left(N^{\prime}[e]\right)$ to at least $k+1$ edges in $P$. Since $|E(G)|=\frac{n r}{2}$ and $|P|=\sum_{e \in E(G)}|S(e) \cap P|$, it follows $(2 r-1)|P|=\sum_{e \in E(G)}\left|S\left(N^{\prime}[e]\right) \cap P\right| \geq \frac{n r}{2}(2 r k-k+1)$. That is, $|P| \geq \frac{n r}{2(2 r-1)} \cdot(2 r k-k+1)$, as required. $\triangle$ 
Corollary 15 The following are equivalent:

(i) $S(G)$ admits a perfect edge dominating set of size $\frac{n r}{2(2 r-1)} \cdot(2 r k-k+1)$

(ii) $G$ can be colored with two colors, $W$ and $Y$, such that

- No two vertices of $W$ are adjacent, and

- Every vertex of $Y$ has exactly one neighbor of the same color in $G$.

(iii) $G$ admits an efficient edge dominating set.

Proof: (i) $\Rightarrow$ (ii)

By the two previous lemmas, whenever $|P|=\frac{n r}{2(2 r-1)} \cdot(2 r k-k+1)$, in each edge neighborhood $N^{\prime}[e]$ of $G$, $2 r-2$ of the edges of $S\left(N^{\prime}[e]\right)$ contain exactly $k$ edges of $P$, while one edge of $N^{\prime}[e]$ contributes with $k+1$ edges to $P$. Color the vertices of $G$, as follows. Assign color $Y$ to both extremes of the edges which contribute with $k+1$ edges and the color $W$ to the remaining vertices of $G$. The coloring so obtained satisfies the required conditions.

(ii) $\Rightarrow$ (i)

By hypothesis, $G$ admits a coloring with colors $W$ and $Y$, satisfying the conditions of the theorem. Such a coloring partitions the edges of $E(G)$ into two types. Those whose extremes are colored $W$ and $Y$, and those with both extremes colored $Y$. If $k=0, G=S(G)$ and we define $P$ as the set of edges with both extremes colored $Y$. If $k \geq 1$ since every yellow vertex has exactly one yellow neighbor in $G$, it follows that, for any $e \in E(G)$, $S\left(N^{\prime}[e]\right)$ contains exactly one edge with both extremes $Y$, while each one of the remaining edges of $S\left(N^{\prime}[e]\right)$ has extremes colored $W$ and $Y$, respectively. We can construct a perfect edge dominating set $P$, by selecting edges from each $N^{\prime}(e)$, where $e=v w \in E(G)$. If $v$ and $w$ are both in $Y$ then $S(e) \cap P=\left\{e_{0}, e_{3}, \ldots, e_{3 k}\right\}$. Otherwise, we can suppose that $v$ is in $W$ while $w$ is in $Y$, and in this case $S(e) \cap P=\left\{e_{1}, e_{4}, \ldots, e_{3 k-2}\right\}$. It is easy to verify that $P$ is a perfect edge dominating set of size $\frac{n r}{2(2 r-1)} \cdot(2 r k-k+1)$.

The proofs (ii) $\Rightarrow$ (iii) and (iii) $\Rightarrow$ (ii) are straightforward. $\triangle$

Next, we present the main result of this section.

Theorem 16 The cardinality perfect edge domination problem is NP-hard, even if restricted to graphs having vertices of bounded degree $r$ and girth at least $k$, for any fixed $r, k \geq 3$.

Proof: It is straightforward to conclude that the problem belongs to $\mathcal{N} P$. The transformation is from the efficient edge domination problem for $r$-regular graphs, which is known to be NP-complete for fixed $r$ (Cardoso, Cerdeira, Delorme and Silva [4). Let $G$ be an $r$-regular graph, $|V(G)|=n$. Construct an instance of the perfect edge domination problem, as follows. Let $k \geq 3$ be a fixed integer, define $k^{\prime}=\max \left\{1,\left\lceil\frac{k-3}{9}\right\rceil\right\}$. The input graph for the latter problem is the $3 k^{\prime}$-subdivision $S(G)$ of $G$. Set the value of $p$, as $p=\frac{n r}{2(2 r-1)} \cdot\left(2 r k^{\prime}-k^{\prime}+1\right)$. Clearly, $S(G)$ has no cycles shorter than $3 \cdot\left(3 k^{\prime}+1\right)=9 k^{\prime}+3 \geq k$ and its vertices have maximum degree $r$. Finally, by Corollary 15, $G$ contains an efficient edge dominating set if and only if $S(G)$ contains a perfect edge dominating set of size $p$ which completes the proof of NP-completeness. $\triangle$

As Lemma 13, Lemma 14 and Corollary 15hold for $k=0$, and using similar arguments of the proof of Theorem 16. we can prove the following corollary.

Corollary 17 The cardinality perfect edge domination problem is NP-hard, even if restricted to r-regular graphs, for $r \geq 3$.

\section{A dichotomy theorem}

In this section, we describe a dichotomy theorem for the complexity of the perfect edge domination problem. It is a consequence of the NP-completeness proofs of the previous sections. 
Define a linear forest as a graph whose connected components are induced paths.

Theorem 18 Let $H$ be a graph, and $\mathcal{G}$ the class of $H$-free graphs of degree at most $d$, for some fixed $d \geq 3$. Then the perfect edge domination problem is

- polynomial time solvable for graphs in $\mathcal{G}$ if $H$ is a linear forest

- NP-complete otherwise.

Proof: First, assume $H$ is not a linear forest. There are two situations. If $H$ contains some induced cycle $C_{s}$ it follows that $\mathcal{G}$ contains, as a subclass, the $C_{s}$-free graphs of maximum degree at most $d$. The latter subclass contains the graphs of bounded degree at most $d$ and girth at least $s+1$. By Theorem 16, the perfect edge dominating set is NP-complete for such a class. For the second alternative, assume that $H$ does not contain cycles. Since $H$ is not a linear forest, it follows that $H$ is a forest containing a vertex of degree at least 3 . That is, $\mathcal{G}$ contains the class of claw-free graphs of maximum degree at most 3. By Corollary 9 the perfect edge domination problem is also NP-complete in this case.

Finally, assume that $H$ is a linear forest and its connected components consist of exactly $t$ disjoint induced paths. In this case, we can bound the number of vertices of each connected component of $G$. Then $H$ is an induced subgraph of $P_{q}$, with $q=|V(H)|+t-1$. An $H$-free graph is in particular $P_{q}$-free, and each connected component of $G$ is $P_{q}$-free and has maximum degree at most $d$. Then each connected component has at most $\frac{d^{q-1}-1}{d-1}$ vertices. That is, a constant number of vertices as $H$ has fixed size. Therefore, we can solve perfect edge domination problem in polynomial time. $\triangle$

Corollary 19 The perfect edge domination problem is NP-complete for $H$-free graphs where $H$ is any graph except linear forests.

We leave as an open problem the question whether or not there exists some graph class for which the efficient edge domination problem is NP-complete and the perfect edge domination problem can be solved in polynomial time.

\section{$7 \quad P_{k}$-free graphs and $P_{5}$-free graphs}

In this section, we describe a robust linear time algorithm for finding minimum weight perfect edge dominating set of a $P_{5}$-free graph. Given an arbitrary graph $G$, in linear time the algorithm either finds such edge dominating set or exhibits an induced $P_{5}$ of the graph.

The following definitions are useful. Let $G$ be a connected graph, and $v \in V(G)$. The eccentricity of $v$, denoted $\epsilon(v)$ is the maximum distance between $v$ and any other vertex. A vertex of minimum eccentricity in the graph is called central. Finally, a vertex having eccentricity at most 2 is named principal vertex.

Note that if a connected graph has a vertex with eccentricity at least 4 then it has an induced $P_{5}$. Any central vertex of a connected $P_{5}$-free graph has eccentricity at most 2 (consequence of Theorem 20).

\subsection{The basis}

We describe below the theorems in which the correctness and complexity of the proposed algorithm is based on.

The following structural result by Bacsó and Tuza is fundamental.

Theorem 20 [1] Every connected graph contains an induced $P_{5}$, a dominating $K_{p}$, or a dominating $P_{3}$. 
The proposed algorithm needs to determine which of these three subgraphs $G$ contains. This will be achieved through a principal vertex. Then we need a robust method to compute such a vertex, if it exists. For a chosen vertex $v$, call Test $(v)$ the operation that determines its eccentricity and recognizes the following situations:

(i) If $v$ has infinite eccentricity, then $G$ is not connected.

(ii) If $v$ has eccentricity at least 4 , then $G$ has an induced $P_{5}$.

(iii) If $v$ has eccentricity 3 , then $G$ has an induced $P_{4}$ starting in $v$,

(iv) If $v$ has eccentricity at most 2 , then $v$ is a principal vertex of $G$.

It is clear that Test $(v)$ can be done in linear-time. The cases (i) and (ii) allow to recognize that the input $G$ is not a connected $P_{5}$-free graph.

The next theorem describes the robust linear time computation of a principal vertex.

Theorem 21 Any connected graph $G$ contains a principal vertex or an induced $P_{5}$. Moreover there is a lineartime algorithm to find a principal vertex of $G$ or to detect that $G$ is not $P_{5}$-free.

Proof: We describe an algorithm that uses at most 3 times the procedure Test a vertex. At any point of the algorithm, if an induced $P_{5}$ is found, then the algorithm ends and returns that induced $P_{5}$. First choose any vertex $u \in V$ and Test $(u)$. If $\epsilon(u)=3$, let $z$ be such that $\operatorname{dist}(u, z)=3$ and $u, v, w, z$ an induced $P_{4}$. Choose $v_{1} \in A=N(u) \cap N(w)$ such that $v_{1}$ has the maximum number of neighbors in $B=V \backslash(N(u) \cup N(w) \cup N(z))$. Note that $u, v_{1}, w, z$ is an induced $P_{4}$ and possibly $v=v_{1}$.

Next, Test $\left(v_{1}\right)$ and Test $(w)$. We will show that $\epsilon\left(v_{1}\right)=\epsilon(w)=3$ implies that $G$ has an induced $P_{5}$. As $v_{1}$ has eccentricity 3 , there exists a vertex $x$ such that $\operatorname{dist}\left(x, v_{1}\right)=3$. Also $x$ verifies $\operatorname{dist}(x, w)=3$ or $\operatorname{dist}(x, w)=2$ because $v_{1} w \in E$.

If $\operatorname{dist}(x, w)=3$ let $v_{1}, a, b, x$ be a shortest path between $v_{1}$ and $x$, as in Figure 5 . Note that $N[x] \cap\left\{u, v_{1}, w, z\right\}=$ $\emptyset, N(b) \cap\left\{v_{1}, w\right\}=\emptyset$ because $\operatorname{dist}\left(x, v_{1}\right)=3$ and $\operatorname{dist}(x, w)=3$. If $N(b) \cap\{u, z\} \neq \emptyset$ there is an induced $P_{5}$ and we can find it and return it. Otherwise, $N(b) \cap\{u, z\}=\emptyset$. We can assume $w a \in E$ otherwise $w, v_{1}, a, b, x$ is an induced $P_{5}$. Following the same idea, $x, b, a, v_{1}, u$ and $x, b, a, w, z$ are possible induced $P_{5}$ 's, then we can assume that $u a, a z \in E$ which is a contradiction because $\operatorname{dist}(u, z)=3$. Therefore, in this case the algorithm always finds an induced $P_{5}$.

If $\operatorname{dist}(x, w)=2$ let $w, a, x$ be a shortest path between $w$ and $x$. Note that $N[x] \cap\left\{u, v_{1}, w\right\}=\emptyset$ because $\operatorname{dist}\left(x, v_{1}\right)=3$. If $z x \in E$ then $u, v_{1}, w, z, x$ is an induced $P_{5}$. Thus, $z x \notin E$ and we are in the situation of Figure 6] We assume $u a \in E$ otherwise $u, v_{1}, w, a, x$ is an induced $P_{5}$. This implies that $a \in A=N(u) \cap N(w)$ and it is adjacent to $x \in B=V \backslash(N(u) \cup N(w) \cup N(z))$. Recall that $v_{1}$ was chosen as a vertex belonging to $A$ with maximum degree in $B$. As $v_{1} x \notin E$, then there exists $y \in B$ such that $v_{1} y \in E$ and $a y \notin E$. It follows that there is a $P_{5}$ induced either by $y, v_{1}, u, a, x$ if $x y \notin E$ or by $x, y, v_{1}, w, z$ if $x y \in E$. Again, the algorithm always finds an induced $P_{5} \cdot \triangle$

Once a principal vertex is obtained, we find an induced $P_{5}$, or a dominating $K_{p}$, or a dominating $P_{3}$ following the theorem below. We remark that using a recent characterization of $P_{k}$-free graphs, by Camby and Schaudt [5], it is possible to obtain a dominating induced $P_{3}$ or a dominating $K_{p}$ in $O\left(n^{5}(n+m)\right)$ time when a connected $P_{5}$-free graph is given.

Theorem 22 For any connected graph $G$, there is a linear-time algorithm to find a dominating induced $P_{3}$, a dominating $K_{p}$, or to detect that $G$ is not a $P_{5}$-free graph.

Proof: The algorithm searches a dominating set contained in $N[v]$ where $v$ is a principal vertex of $G$. Again, at any point of the algorithm, if an induced $P_{5}$ is found, then the algorithm ends and returns that induced $P_{5}$. 


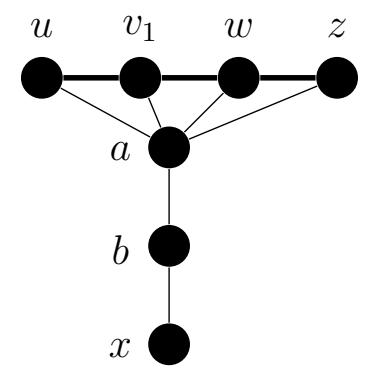

Figure 5: $\operatorname{dist}\left(v_{1}, x\right)=\operatorname{dist}(w, x)=3$.

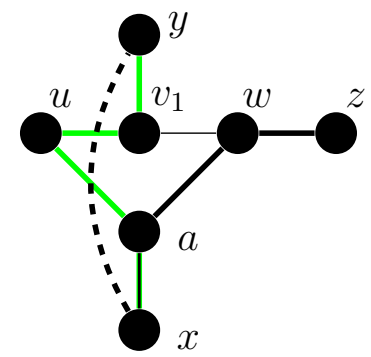

Figure 6: $\operatorname{dist}\left(v_{1}, x\right)=3$ and $\operatorname{dist}(w, x)=2$.

1. Find a principal vertex $v$ or an induced $P_{5}$ of $G$ using the robust linear-time algorithm of Theorem 21

2. Let $X:=N[v]$ be the initial dominating set. Consider iteratively each vertex $w \in N(v)$ : if $X \backslash\{w\}$ is still a dominating set then $X:=X \backslash\{w\}$. This can be done in linear-time using a variable for each vertex of $V \backslash N[v]$ to count the number of neighbors in $X$. A vertex $w \in N(v)$ can not be removed from $X$ if only if some of its neighbors has exactly one dominator in $X$.

3. If $|X| \leq 3$ then $G$ has a dominating $P_{3}$ or a dominating $K_{|X|}$. If $|X| \geq 4$ and it is not a complete graph, we will show that $G$ has an induced $P_{5}$. We can assume that $G$ has a subgraph like one in Figure 7. where $\left\{v_{1}, v_{2}, v_{3}\right\} \subset X$ and does not induce a triangle. Each one of them has a proper dominated vertex $w_{1}, w_{2}, w_{3}$ respectively. Edges between $w_{i}$ and $w_{j}$ are drawn in order to avoid the induced $P_{5}$ $w_{i}, v_{i}, v, v_{j}, w_{j}$. Nevertheless in (i) and (ii) $w_{2}, w_{1}, v_{1}, v, w_{3}$ is a induced $P_{5}$; and in (iii) the vertices $w_{2}, v_{2}, v_{3}, w_{3}, w_{1}$ or $w_{2}, w_{1}, v_{1}, v, w_{3}$ induce a $P_{5}$.

$\triangle$

We remark that the above theorem might be of interest to other $P_{5}$-free algorithmic problems, since it represents an algorithmic proof of Bacsó and Tuza's theorem [1].

(i)

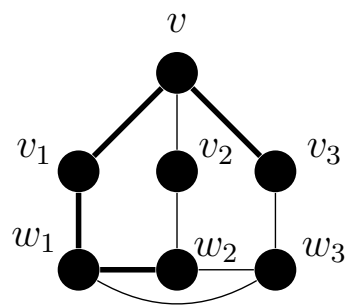

(ii)

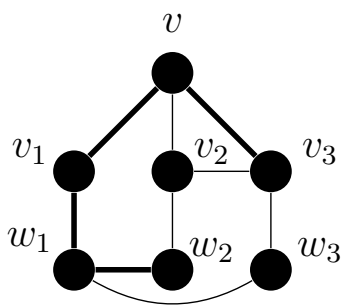

(iii)

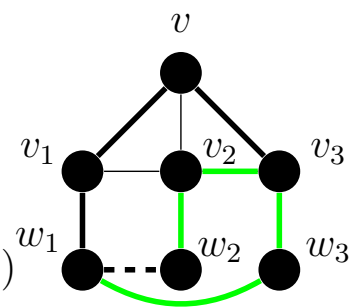

Figure 7: Center $v$ and its sons and proper grandsons. 


\subsection{Colorings, vertex dominating sets and perfect edge dominating sets}

Next, we describe the remaining algorithms on which the proposed solution is based. They are related to 3colorings of the graph, vertex dominating sets and the different kinds of perfect edge dominating sets, that is, efficient edge dominating, trivial ones and proper.

The first two theorems concern the construction of perfect edge dominating sets, associated to a given 3-coloring of the graph.

Theorem 23 Given a graph $G$ and $W \subseteq V$, there is a linear-time algorithm to verify if there exists some perfect edge dominating set whose associated 3-coloring is $(B, Y, W)$.

Proof: In the affirmative case, using Observation 2, we can determine $Y$ in linear-time and so $B=V \backslash(W \cup Y)$. Therefore, we construct a 3 -coloring $(B, Y, W)$ in this way and then test the validity of $(B, Y, W)$ by checking the conditions of Observations 1, 2 and 3. All these computations can be done in linear-time. $\triangle$

Theorem 24 Given a connected graph $G$ and $Y \subset V$, there is a linear-time algorithm to verify if there exists some perfect edge dominating set whose associated 3-coloring is $(B=\{b\}, Y, W)$. Moreover, the algorithm can find a vertex $b$ for which the sum of the weight of its incident edges is minimum.

Proof: Clearly, if such perfect edge dominating set exists, it verifies

i. $G[Y]$ is a graph with maximum degree 1 and at least one vertex has degree 0 .

ii. $V \backslash Y$ is a independent set.

iii. If $\left\{s_{1}, s_{2}, \ldots, s_{k}\right\} \subseteq Y$ are the vertices with degree 0 in $G[Y]$, and $\left\{y_{1}, y_{2}, \ldots, y_{l}\right\} \subset Y$ are the vertices of degree 1 , the black vertex belongs to $\bigcap_{i=1}^{k} N\left(s_{i}\right) \backslash \bigcup_{j=1}^{l} N\left(y_{j}\right)$.

Therefore, we check conditions (i) and (ii), and construct $A=\bigcap_{i=1}^{k} N\left(s_{i}\right) \backslash \bigcup_{j=1}^{l} N\left(y_{j}\right)$ in linear-time. Note that all vertices in $A$ are equivalent. If $A \neq \emptyset$, each $b \in A$ generates a valid coloring $(B, Y, W), B=\{b\}, W=$ $V \backslash(B \cup Y)$. Condition (ii) and $|B|=1$ imply that $(B, Y, W)$ satisfies the conditions of Observations 1 and 3. Condition (i) and $b \in A$ imply that $(B, Y, W)$ satisfies the condition of Observation 2 , So, the validity of $(B, Y, W)$ holds. $\triangle$

The next theorem refers to finding an efficient edge dominating set of a graph, given a fixed size vertex dominating set of it.

Theorem 25 [11] Given a graph $G$ and a vertex dominating set of fixed size of $G$, there is a linear-time algorithm to solve the minimum weight efficient edge domination problem for $G$.

The following results relate trivial perfect edge dominating sets of a graph and the existence of vertex dominating complete subgraphs of certain sizes.

Observation 26 Given a graph $G$, a dominating set $D$ of $G$, a perfect edge dominating set $P$ of $G$ and $(B, Y, W)$ the 3-coloring associated to $P$, if $D \subseteq B$ then $P$ is the trivial perfect edge dominating set.

This observation is a direct consequence of Observation 3 .

Corollary 27 Given a connected graph $G$, if there is a dominating $K_{p}$ with $p \geq 4$ then $G$ has exactly one perfect edge dominating set $P$ and $P$ is trivial. 
Proof: Suppose that there is a non-trivial perfect edge dominating set $P$. By Observation 4 , all vertices of $K_{p}$ must be black and by Observation 26, $P$ must be trivial and this is a contradiction. $\triangle$

Corollary 28 Given a connected $P_{5}$-free graph $G$, if $G$ admits some non-trivial perfect edge dominating set $P$ then $G$ has dominating induced $P_{3}$ or a dominating $K_{3}$.

Proof: If $G$ has at most 2 vertices, then $G$ has only one perfect edge dominating set which is trivial. Therefore, $G$ has at least 3 vertices. Now, suppose that $G$ has neither dominating induced $P_{3}$ nor dominating $K_{3}$. By Theorem 20, $G$ must have some dominating $K_{p}$ with $p \neq 3$. In case $p \geq 4$, by Corollary 27, $G$ has no non-trivial perfect edge dominating set which is a contradiction. Therefore, $p \leq 2$. As $G$ is connected with at least 3 vertices, it is always possible to add more vertices to $K_{p}$ to form a dominating $K_{3}$ or a dominating induced $P_{3}$. Again, a contradiction. $\triangle$

Corollary 29 Given a graph $G$, if $G$ has some vertex dominating $K_{1}$ or $K_{3}$ then $G$ has no proper edge dominating sets.

Proof: First, assume $G$ has a dominating $K_{1}=\{u\}$ which means that $u$ is a universal vertex. Suppose that $P$ is a proper edge dominating set. Let $v$ be any black vertex. By Observation 26, $v$ cannot be a universal vertex. As $u$ is a neighbor of $v$, then $u$ is a yellow vertex because it is a universal vertex. As $v$ is a black vertex, $v$ has another non-white neighbor $w$. Moreover, $w$ is adjacent to the universal vertex $u$. This contradicts that $u$ is a yellow vertex.

Next, suppose $G$ contains a dominating $K_{3}$. Suppose that $G$ has some proper edge dominating set $P$ and $(B, Y, W)$, its associated 3-coloring, with $B, Y, W \neq \emptyset$. By Observation 5 , (i) all vertices of the dominating $K_{3}$ are black or (ii) exactly two of them are yellow vertices and the other vertex is white. In case (i), by Observation 26, $P$ must be trivial which is a contradiction. In case (ii), there are no black vertices as consequence of Observations 2 and 3 . Again, a contradiction. $\triangle$

Finally, the last theorem relates the existence of proper edge dominating sets to special colorings of a dominating $P_{3}$.

Theorem 30 Given a connected $P_{5}$-free graph $G$, if $G$ admits some proper perfect edge dominating set $P$, with associated 3-coloring $(B, Y, W)$, then $G$ has a vertex dominating $P_{3}$ (formed by vertices $v_{1}, v_{2}$ and $v_{3}$ ) with one of the possible combinations of colors of Figure 8 .

Proof: Clearly, by Corollaries 28 and $29, G$ has a dominating induced $P_{3}$. By Observations 1 , 2] and 3 , there exists neither two adjacent white vertices, nor yellow vertices with at least two non-white neighbors nor white vertices with black neighbors. As a consequence, the possible colors of the dominating induced $P_{3}$ must match to some combinations of Figure 8 or some of Figure 9] If the combination (f) of Figure 9 is matched then all vertices of the dominating induced $P_{3}$ are black vertices and by Observation 26, $P$ is a trivial perfect edge dominating set leading to a contradiction. If the combination ( $\mathrm{g}$ ) of Figure 9 is matched, two adjacent vertices of the dominating induced $P_{3}$ are yellow vertices and the other one is a white vertex. None of these three vertices can have black neighbors which implies $B=\emptyset$ and $P$ to be an efficient dominating set. Again, this is a contradiction. Therefore, the only valid options are those of Figure $8 \triangle$

\subsection{The algorithm}

Let $G,|V(G)|>1$, be an arbitrary connected graph. The proposed algorithm, along the process, constructs a set $\mathcal{E}$ containing a few candidates for the minimum weight perfect edge dominating set of $G$. At the end, it selects the least of them and returns the minimum edge dominating set of $G$, 
(a)

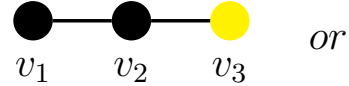

(b)
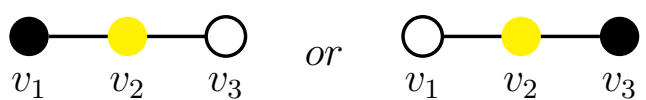

(c)

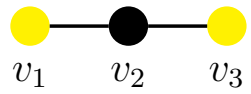

(d)

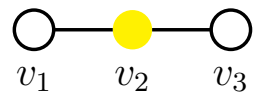

$(e)$

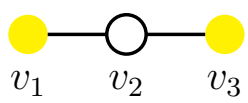

Figure 8: Possible valid colorings of dominating induced $P_{3}$

$(f)$

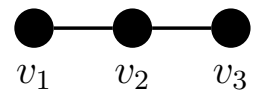

(g)

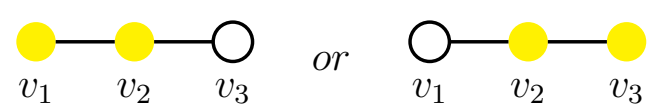

Figure 9: Invalid colorings of dominating induced $P_{3}$ 
1. Define $\mathcal{E}:=\{E(G)\}$

2. Find a principal vertex of $G$. If no such vertex exists then return an induced $P_{5}$ and stop.

3. Using the principal vertex $v$, find (i) an induced $P_{5}$, or (ii) a dominating $K_{p}$, or (iii) a dominating $P_{3}$.

4. Case (i): An induced $P_{5}$ is found. Then return it and stop.

5. Case (ii): A dominating $K_{p}$ is found. If $p \geq 4$ then return $E(G)$ and stop. Otherwise, $p \leq 3$ and using such dominating $K_{p}$, find a minimum weight efficient edge dominating set and, if it exists, include it in $\mathcal{E}$. If $K_{p}=\left\{v_{1}, v_{2}\right\}$ and $N\left(v_{1}\right) \cap N\left(v_{2}\right)=\emptyset$ then transform the dominating $K_{2}$ into a dominating $P_{3}$ by adding a third vertex to it.

6. Case (iii): A dominating $P_{3}$ is found. First, again using such a vertex dominating set, find a minimum weight efficient edge dominating set of $G$, and include it in $\mathcal{E}$, if it exists. Then look for a proper perfect edge dominating set of $G$, by considering every possible coloring of the $P_{3}$, according to Figure 8 , In each of the cases (a)-(b) ((c)-(e)), below, the algorithm determines at most two (one) proper perfect edge dominating sets (set), which are (is) then included in $\mathcal{E}$.

(a) Without loss of generality, $v_{3}$ is the yellow vertex of the dominating induced $P_{3}$. Clearly, $W=$ $N\left(v_{3}\right) \backslash\left\{v_{2}\right\}$ and we can apply the linear-time algorithm of Theorem 23 to determine the proper perfect edge dominating set whose associated 3-coloring is $(B, Y, W)$. Include it in $\mathcal{E}$, if it exists.

(b) Without loss of generality, $v_{1}$ is the black vertex of the dominating induced $P_{3}$. We can applied the same technique of (a) using $W=N\left(v_{2}\right) \backslash\left\{v_{1}\right\}$.

(c) Again, apply the same technique of (a) using $W=\left(N\left(v_{1}\right) \cup N\left(v_{3}\right)\right) \backslash\left\{v_{2}\right\}$.

(d) In this case, $v_{1}$ and $v_{3}$ have only yellow neighbors. As we are looking for proper perfect edge dominating sets, there is some black vertex somewhere. Clearly, $v_{2}$ must have exactly one black neighbor and the other neighbors of $v_{2}$ are white vertices. So, $Y=N\left(v_{1}\right) \cup N\left(v_{3}\right),|B|=1$, and we can apply the linear-time algorithm of Theorem 24 to determine the proper perfect edge dominating set whose associated 3-coloring is $(B, Y, W)$ with least weight. Again, if successful include it in $\mathcal{E}$.

(e) In this case, $v_{1}\left(v_{3}\right)$ can have at most one black neighbor. As we are looking for proper perfect edge dominating sets, there is at least one black vertex. First, we assume that there are some triangles using edges of the dominating induced $P_{3}$ (the number of these triangles is exactly $\left|N\left(v_{1}\right) \cap N\left(v_{2}\right)\right|+$ $\left|N\left(v_{2}\right) \cap N\left(v_{3}\right)\right|$ which can be computed in linear-time). If there are at least 2 triangles using the same edge, without loss of generality $\left\{v_{1}, v_{2}, x\right\}$ and $\left\{v_{1}, v_{2}, x^{\prime}\right\}$, then the coloring is invalid. If $\left\{v_{1}, v_{2}, x\right\}$ and $\left\{v_{2}, v_{3}, x^{\prime}\right\}$ are triangles then the coloring is invalid or it cannot admit black vertices, which means there are no proper perfect edge dominating sets. The only possibility for the graph to admit a proper perfect edge dominating set (which implies the existence of black vertices) is the existence of exactly one triangle using an edge of the dominating induced $P_{3}$ and $|B|=1$. Clearly, the yellow vertices are $Y=N\left(v_{2}\right)$ and we then proceed as in Case (d). Next, suppose there is no triangle using an edge of the dominating induced $P_{3}$. Clearly, every vertex in $A_{1,3}=N\left(v_{1}\right) \backslash N\left(v_{3}\right)$ has to be adjacent to every vertex in $A_{3,1}=N\left(v_{3}\right) \backslash N\left(v_{1}\right)$ or there is some induced $P_{5}$, which the algorithm returns and then stops. It is not hard to see this can be accomplished in linear-time. Suppose that there is only a black vertex $b$. It has to be adjacent to $v_{1}$ and $v_{3}$, otherwise (without loss of generality, $\left.b v_{1} \notin E(G)\right)$ there is an induced $P_{5}$ formed by $y, v_{1}, v_{2}, v_{3}, b$ where $y$ is a yellow neighbor of $v_{1}$. Then the yellow vertices are $Y=N\left(v_{2}\right)$ and we proceed as in $(\mathrm{d})$. The last case is that there are two black vertices $b_{1}, b_{3}$ where $b_{1} \in A_{1,3}$ and $b_{3} \in A_{3,1}$, and the coloring is invalid if $\left|A_{1,3}\right|>1$ or $\left|A_{3,1}\right|>1$. The algorithm will check if $\left(B=\left\{b_{1}, b_{3}\right\}, Y=N\left(v_{2}\right), W=V \backslash(B \cup Y)\right)$ is a valid coloring, and include in $\mathcal{E}$ the corresponding edge dominating set. All these computations can be done in linear-time.

7. Select the least weight perfect edge dominating set of $\mathcal{E}$, return it and stop.

The correctness and linear time complexity of the algorithm follows directly from the propositions formulated in the previous subsections.

To summarise we state the main result of this section. 
Theorem 31 The weighted perfect edge domination problem can be solved for $P_{5}$-free graphs in linear time in a robust way.

\section{References}

[1] G. Bacsó, Zs. Tuza, Dominating cliques in $P_{5}$-free graphs, Periodica Mathemayica Hungarica 21, 303-308 (1990).

[2] A. Brandstadt, A. Leitert, D. Rautenbach, Efficient dominating and edge dominating sets for graphs and hypergraphs, Proceedings of the 23rd International Symposium on Algorithms and Computation (ISAAC 2012), 2012, Lecture Notes in Computer Science, 7676, 558-277 (2012).

[3] A. Brandstadt, R. Mosca, Dominating induced matching for $P_{7}$-free graphs in linear time. Proceedings of the 22nd International Symposium on Algorithms and Computation (ISAAC 2011), Lecture Notes in Computer Science, 100-109 (2011).

[4] D. M. Cardoso, J. O. Cerdeira, C. Delorme, P. C. Silva, Efficient edge domination in regular graphs, Discrete Applied Mathematics 156, 3060-3065 (2008).

[5] E. Camby, O. Schaudt, A new characterization of $P_{k}$-free graphs, Graph-Theoretic Concepts in Computer Science - 40th International Workshop (WG 2014), France, Revised Selected Papers, 129-138 (2014).

[6] D. M. Cardoso, N. Koperlainen, V. V. Lozin, On the complexity of the induced matching problem in hereditary classes of graphs, Discrete Applied Mathematics 159, 521-531 (2011).

[7] J. P. Georges, M. D. Halsey, A. M. Sanaulla, M. A. Whittlesey, Edge domination and graph structure, Congressus Numerantium 76, 127-144 (1990).

[8] D. L. Grinstead, P. J. Slater, N. A. Sherwani, N. D. Holnes, Efficient edge domination problems in graphs, Informatio Processing Letters 48, 221-228 (1993).

[9] A. Hertz, V. Lozin, B. Ries, V. Zamaraev, D. de Werra, Dominating induced matchings in graphs containing no long claw, arXiv:1505.02558.

[10] M. C. Lin, M. Mizrahi, J. L. Szwarcfiter, An $O^{*}\left(1.1939^{n}\right)$ time algorithm for minimum weighted dominating induced matching Proceedings of the 24th International Symposium on Algorithms and Computation (ISAAC 2013), 2013, Hong Kong, Lecture Notes in Computer Science, 8283, 558-567 (2013).

[11] M. C. Lin, M. Mizrahi, J. L. Szwarcfiter, Exact algorithms for dominating induced matching. Corr, abs/1301.7602, (2013).

[12] M. C. Lin, M. Mizrahi, J. L. Szwarcfiter, Fast algorithms for some dominating induced matching problems, Information Processing Letters 114, 524-528 (2014).

[13] M. C. Lin, M. Mizrahi, J. L. Szwarcfiter, Efficient and perfect domination on circular-arc graphs, Proceedings of the VIII Latin-American Graphs, Algorithms and Optimization Symposium (LAGOS' 2015), Beberibe, Brazil, Electronic Notes in Discrete Mathematics, to appear (2015).

[14] M. C. Lin, V. Moyano, D. Rautenbach, J. L. Szwarcfiter, The Maximum Number of Dominating Induced Matchings, Journal of Graph Theory 78, 258-268 (2015).

[15] C. L. Lu, M.-T. Ko and C. Y. Tang, Perfect edge domination and efficient edge domination in graphs, Discrete Applied Mathematics 119, 227-250 (2002).

[16] C. L. Lu, C. Y. Tang, Solving the weighted efficient edge domination problem in bipartite permutation graphs, Discrete Applied Mathematics 87, 203-211 (1998).

[17] M. Xiao, H. Nagamochi, Exact algorithms for dominating induced matching based on graph partition, Discrete Applied Mathematics 190-191, 147-162 (2015). 